\title{
ONDA DE FRIO? ANÁLISE DE DIFERENTES MÉTODOS DE IDENTIFICAÇÃO
}

\author{
ALVES, Maikon Passos Amilton - maiconpassos@gmail.com \\ Universidade Federal de Santa Catarina/UFSC \\ SILVEIRA, Rafael Brito - rafael.brito@posgrad.ufsc.br \\ Universidade Federal de Santa Catarina/UFSC
}
BITENCOURT, Daniel Pires - daniel.bitencourt@fundacentro.gov.br
Fundação Jorge Duprat Figueiredo de Segurança e Medicina do
Trabalho/FUNDACENTRO

FRANKE, Alberto Elvino - alberto.franke@ufsc.br
Universidade Federal de Santa Catarina/UFSC

\begin{abstract}
RESUMO: Episódios de extremos de temperatura, por vezes, são negligenciados como desastres no Brasil. Maior atenção é dada quando são gerados prejuízos para a agricultura, entretanto, os extremos de temperatura ou as ondas de frio (OdF) e as ondas de calor também impactam em outros setores, sem hierarquizá-los quanto a importância. Por mais que uma vertente de climatólogos (talvez a mais evidente) aponte para o aquecimento, de forma geral, da Terra e, seja dada mais importância aos futuros eventos de calor; a maioria das pesquisas aponta para uma intensificação dos eventos extremos, inclusive os de frio. Neste sentido, o presente artigo tem como objetivo central, analisar dez métodos distintos para identificação de OdF, testando-os e comparando-os entre si, em três locais diferentes de Santa Catarina (Lages, Chapecó e Florianópolis), a partir de dados observados de estações convencionais entre 2000 e 2010. Os resultados do número $\left(n^{\circ}\right)$ de OdF detectados pelos dez métodos, por estação do ano, nas três localidades, foram submetidos à Análise da Variância Fatorial com nível de significância de $a=5 \%$, para verificar quais métodos são diferentes estatisticamente entre si. Posteriormente, foi aplicado o teste de comparação múltipla de médias de ocorrências de OdF pelo teste Tukey ao nível de $a=5 \%$. Todas as análises foram realizadas no software STATISTICA $8.0 \AA$. Os resultados principais demonstraram que os métodos de detecção de OdF variaram, estatisticamente, em função do local e da época do ano, apresentando interação com as estações anuais. O rigor específico de cada método é determinante para o $\mathrm{n}^{\circ}$ de OdF, baseado nos limiares estabelecidos e também no $\mathrm{n}^{\circ}$ de dias. Alguns métodos se destacaram pelo grande rigor na identificação das ondas, outros pelo menor rigor, refletindo no $n^{\circ}$ total; aqueles que se utilizam da temperatura média (Tméd) foram os intermediários, com a utilização da Tméd espera-se eliminar problemas com locais frios apenas em um momento do dia (amplitude térmica). Por fim, entende-se que a determinação de qual método utilizar depende da finalidade do estudo e, necessariamente, é preciso buscar uma padronização em nível nacional.
\end{abstract}

Palavras-chaves: Onda de frio, Métodos distintos, Análise estatística, Santa Catarina.

\section{COLD WAVE? ANALYSIS OF DIFFERENT IDENTIFICATION METHODS}

ABSTRACT: In Brazil, extreme temperature events are sometimes neglected as disasters. Greater attention is devoted when agricultural losses are incurred; however, extreme temperatures or cold waves and heat waves also affect other sectors, and no hierarchy of importance is arranged. Although some climatology research strands (perhaps the one most in evidence) tackle global warming in a general way and assign greater importance to future heat events, most studies point to an intensification of extreme events, including cold ones. In this regard, this paper aims to analyze ten 
different methods of cold wave identification by testing and comparing them to each other, in three cities in the state of Santa Catarina (Lages, Chapecó and Florianópolis), Brazil, with data obtained from observations at conventional weather stations between years 2000 and 2010. The data with the number of cold waves detected by the ten methods, by season of the year, in the three cities, underwent factorial Analysis of Variance with significance level of $a=0.05$ in order to verify which methods are statistically different from each other. Subsequently, multiple comparisons of mean cold wave occurrence were carried out by the Tukey test at the significance level of $a=0.05$. All analyses were performed in the STATISTICA $8.0 \AA$ software. The main results demonstrated that the cold wave identification methods varied statistically according to location and time of year, showing interaction with the seasons. The specific rigor of each method is critical in determining the number of cold waves, drawing on the established thresholds and also on the number of days. Some methods stood out for their great rigor while others for being less rigorous, thus influencing the overall number. The methods using mean temperatures were intermediate - the use of mean temperatures aims to eliminate problems with places that are cold only at a certain time of the day (thermal amplitude). Finally, it was understood that determining a suitable method depends on the purpose of each study and, necessarily, a standardization should be sought at the national level.

Keywords: Cold wave, Different methods, Statistical analysis, Santa Catarina.

\section{INTRODUÇÃO}

O frio em Santa Catarina (SC) é uma condição presente todos os anos, por mais que varie de intensidade ano a ano e ao longo do território. Quando comparamos a Região Sul às demais do país, observamos que os episódios impactantes de frio são mais recorrentes, especialmente quando ligados aos locais com altitudes mais elevadas. Nas maiores altitudes de SC, as temperaturas podem ser negativas, influenciando na formação de geada ou neve, sob ocorrência de massa de ar frio, especialmente durante o inverno. No estado catarinense as temperaturas tendem a aumentar no sentido sul-norte e das áreas mais elevadas do planalto para oeste e leste (MONTEIRO; MENDONÇA, 2014, p.6). Monteiro (1963) já apontava para os fatores geográficos que conferem à porção extratropical da América do Sul a característica climática subtropical, que diante do quadro térmico geral do Brasil faz com que existam temperaturas consideravelmente baixas.

A latitude do estado faz com que ele esteja submetido a muitos sistemas atmosféricos que atuam no Brasil ao longo do ano. Quando uma massa de ar frio avança sobre o sul brasileiro, a mesma ocasiona ventos fortes no quadrante sul e queda significativa na temperatura do ar em todas as regiões (MONTEIRO; MENDONÇA, 2014, p.6). Nos meses de inverno os sistemas atmosféricos que ocorrem com maior frequência no estado são os frontais. Estes, em outras épocas do ano, também podem condicionar temperaturas abaixo da média esperada para uma determinada estação, pois, as massas de ar polar Atlântica ( $\mathrm{mPa}$ ) que o sucedem, atuam com grande frequência e intensidade, sendo responsáveis pelos episódios de menores temperaturas registradas, causando até neve (MURARA; FUENTES, 2014, p.164). Segundo Borsato (2016, p.101) durante o inverno as massas frias se intensificam e avançam com maior frequência pela porção interior do continente sul-americano; para os meses mais quentes as massas polares se deslocam de forma mais interiorana pelo Atlântico e raramente pelo interior do continente, todavia, esta dinâmica para o sul da América do Sul é constante e ocorre em todas as estações do ano. 
De março em diante, especialmente no trimestre invernal, as massas de ar quente encontram-se deslocadas para regiões com latitudes mais baixas, em decorrência as temperaturas são menores em SC, devido principalmente às incursões de massas de ar polares migratórias, que com o resfriamento do continente, adentram de forma mais dominante (NIMER, 1979; MONTEIRO, 2001; ESCOBAR, 2007; MENDONÇA; ROMERO, 2012).

Conforme Silveira RB (2016), o Brasil diante de parcela da população latino-americana e até mundial, é visto como um país tropical em sua totalidade, todavia, parte do território brasileiro está localizado na porção subtropical do hemisfério sul. Tal especificidade é responsável por algumas características distintas de variáveis ambientais se comparado ao Brasil tropical. Este entendimento de que o território brasileiro é totalmente tropical, muitas vezes faz com que as pessoas sem o conhecimento necessário pensem que as condições de tempo e clima no Brasil não sejam submetidas a ondas de frio (OdF), característica peculiar de épocas do ano na porção subtropical do globo, especialmente se está atrelada a altas altitudes. Mendonça e Romero (2012) constataram que as OdF por vezes provocam enfermidades e mortes de pessoas e perdas econômicas, impactando no desenvolvimento das atividades na cidade e no campo, danificando paisagens e culturas em diversos países da América do Sul.

Condições geográficas locais não explicam o tempo e o clima por si só, os sistemas atmosféricos possuem participação como condicionante nas baixas temperaturas catarinenses e em outras variáveis climáticas, mostrando assim a necessidade de uma análise baseada na multiescalaridade para explicar eventos locais e regionais.

Todos aqueles que pesquisam sobre OdF e onda de calor (OdC) se deparam com diversos métodos de identificação destes eventos, sejam eles em nível local, regional, nacional e até internacional. A divergência e a pluralidade de métodos podem dificultar o estudo ou ainda confundir a interpretação dos resultados, pois diferentes métodos, possivelmente, apresentarão conclusões distintas em um mesmo local ou domínio. Segundo o 50 Assessment Report, do Intergovernamental Panel on Climate Change (IPCC), ao longo do século XXI, é muito provável que as temperaturas altas sejam mais frequentes e que os extremos frios ocorram em menor número $\left(n^{\circ}\right)$ na maioria dos continentes, especialmente pelo aumento da temperatura na Terra. Entretanto, mesmo com este possível aquecimento, os extremos de frio ocasionais continuarão a ocorrer, com mais evidência no inverno. A menor ocorrência de dias e noites frias na maioria das áreas continentais é tida como "virtualmente certa" para o final deste século (IPCC, 2013, p. 7, 20). Todavia, os extremos de frio permanecerão afetando em diferentes setores da sociedade, seja na agropecuária, turismo e/ou na saúde humana (CARSON et al., 2006; MORABITO, 2006; MURARA et al., 2010; MATEUS, 2014; ALVES et al., 2015; SILVEIRA et al., 2015; FRANKE et al., 2015; GASPARRINI et al., 2015; TOBÍAS et al., 2017).

As ondas de frio também são eventos extremos que devem ser considerados desastres, inclusive sendo classificado pelo Manual de Desastres Naturais do Ministério da Integração do Brasil, organizado por Castro et al. (2003). Conforme o Centre for Research on the Epidemiology of Disasters (CRED) (2017), por meio do International Disasters Database (EM-DAT), entre 
1900 e 2016 ocorreram 289 OdF em todo globo. Estas ondas já provocaram 17.367 óbitos, afetando 14.441 .421 pessoas e com danos estimados de, aproximadamente, 17 bilhões de dólares (CRED/EM-DAT, 2017). Por mais que existam esforços para agrupar tais informações em bancos de dados únicos, como o CRED/EM-DAT tem realizado, ainda assim existem subnotificações, pois conforme Silveira et al. (2017) não há integração total das bases nacionais e internacionais. Além disso, a forma como o desastre ou o evento é notificado varia de acordo com os parâmetros estipulados para tal, pois podem partir do impacto para classificar a OdF como desastre ou da análise atmosférica a fim de relacioná-los com possíveis problemas socioambientais (SILVEIRA et al., 2017, p. 50).

Diferentes autores e organizações já elaboraram métodos para identificação de OdF, em alguns casos, baseados na realidade em que estavam inseridos (municípios, estados, regiões), em outros para abranger maiores recortes espaciais. Outros utilizam tais métodos em aplicações específicas, por vezes, com adaptações (REBOITA et al., 2015; ALVES, 2016; SILVEIRA RB, 2016; ALVES et al., 2017), objetivando analisar os impactos das OdF na agricultura, pecuária, saúde das populações, entre outras. Em alguns casos, estes impactos são positivos, beneficiando setores como o turismo, por exemplo. O fato é que métodos distintos podem apresentar resultados divergentes, influenciando diretamente qualquer tipo de análise socioambiental.

Por conta disto, o objetivo desse artigo foi analisar dez métodos diferentes de identificação de OdF, testando-os e comparando-os entre si, em três localidades distintas de Santa Catarina. Essas localidades foram selecionadas por possuírem características climáticas diferentes entre si, possibilitando assim auxiliar na escolha do método mais adequado em futuros estudos.

\section{METODOLOGIA E DADOS}

\section{DADOS DE TEMPERATURA}

Os dados de temperatura foram coletados a partir de observações realizadas nas estações meteorológicas convencionais de Florianópolis (São José), Lages e Chapecó, no período de 2000 a 2010 (11 anos), consistindo em uma série de dados homogênea (mesmo período para as três localidades). São registros diários, fornecidos pelo Instituto Nacional de Meteorologia (INMET), com acesso através do Banco de Dados Meteorológicos para Ensino e Pesquisa (BDMEP) e; também pelo Centro de Informações de Recursos Ambientais e de Hidrometeorologia de Santa Catarina (CIRAM), pertencente à Empresa de Pesquisa Agropecuária e Extensão Rural de Santa Catarina (EPAGRI).

A porcentagem de falhas (ausência de dados) para Florianópolis não ultrapassou $0,15 \%$ para a Tmín, Tmáx e Tméd e, foi de $0,17 \%$ no período observado (2000-2010), dados utilizados de acordo com o método de Firpo et al. (2012); diante de toda série histórica da Tmín desta estação as falhas ficaram abaixo de 1,5\%. Para Chapecó a porcentagem de falhas foi de 1,99\% na Tmín, $1,86 \%$ na Tmáx, $4,45 \%$ na Tméd e, $1,86 \%$ no período observado para o método de Firpo et al. (2012); para toda série histórica da Tmín desta estação as falhas foram de $14,77 \%$. Em Lages a porcentagem de falhas ficou 
assim distribuída: sem falhas para Tmín, 0,02\% na Tmáx, 0,04\% na Tméd e, diante do método de Firpo et al. (2012) para o período observado não existiram falhas, contudo; ao longo de toda série histórica desta estação para a Tmín as falhas foram de 9,37\%. Optou-se por não tratar as falhas devido ao fato de serem poucas e não comprometerem as análises de forma geral. Certamente se não existissem falhas, as análises seriam mais apuradas, especialmente para as análises utilizando o método de Firpo et al. (2012), todavia, o preenchimento foi desconsiderado como a melhor solução, principalmente por se tratarem de dados de temperatura do ar.

\section{OS MÉTODOS TESTADOS}

Os dez métodos avaliados neste estudo divergem principalmente pela variável selecionada para identificação da OdF (Quadro 1). Alguns utilizam dados de temperatura mínima do ar (Tmín), por exemplo: EE - Silveira (2016); Índice Australiano; Cold Spell Duration Index (CSDI-OMM); OMM; Indice do IPCC; Firpo et al. (2012) e Percentil 5. Outros utilizam da Tmín em associação com a temperatura máxima (Tmáx), como: Tmáx e Tmín (10\%) e; outros ainda baseiam-se na temperatura média (Tméd) do ar, por exemplo: Nairn e Fawcett (2013) e Vavrus et al. (2006). Além disso, há ainda muitas distinções na aplicação dessas variáveis, conforme sintetizado no Quadro 1. A característica do método, apresentada ao lado da variável, foi mensurada com base na duração mais o rigor estatístico.

Quadro 1 - Métodos analisados na pesquisa, publicações que se utilizaram dos mesmos, característica do rigor e suas respectivas descrições para identificação das OdF.

\begin{tabular}{|c|c|c|c|}
\hline Método & Publicações base e outras & Variável/Característica & Descrição do método \\
\hline $\begin{array}{l}\text { EE - Silveira } \\
\quad(2016) \\
\text { adaptado }\end{array}$ & Silveira RD (2016) & $T_{\min }$ do ar / Ameno & $\begin{array}{c}\text { Pelo menos } 3 \text { dias consecutivos } \\
\text { com temperatura mínima } \\
\text { abaixo do percentil } 10 \text { de cada } \\
\text { estação do ano correspondente } \\
\text { ao período. }\end{array}$ \\
\hline $\begin{array}{c}\text { Índice } \\
\text { Australiano }\end{array}$ & $\begin{array}{l}\text { Bureau of Meteorology } \\
(2017)\end{array}$ & $T_{\min }$ do ar / Rigoroso & $\begin{array}{l}\text { Pelo menos } 4 \text { dias consecutivos } \\
\text { com a temperatura mínima } \\
\text { inferior ao percentil } 10 \\
\text { encontrado para aquela estação } \\
\text { do ano correspondente ao dia. }\end{array}$ \\
\hline CSDI - OMM & OMM (2011) & $\begin{array}{l}T_{\min } \text { do ar / Muito } \\
\text { rigoroso }\end{array}$ & $\begin{array}{l}\text { Pelo menos } 6 \text { dias consecutivos } \\
\text { com temperaturas mínimas } \\
\text { inferiores ao percentil } 10 \text { de } \\
\text { cada estação do ano } \\
\text { correspondente ao dia. }\end{array}$ \\
\hline $\begin{array}{l}T_{\text {máx }} \text { e } T_{\min } \\
(10 \%)- \\
\text { adaptado }\end{array}$ & $\begin{array}{c}\text { Yague et al. (2006); } \\
\text { Landa (2012); Silveira RD } \\
\text { (2016) }\end{array}$ & $\begin{array}{l}T_{\min } \text { e } T_{\operatorname{máx}} \text { do ar / } \\
\text { Rigoroso }\end{array}$ & $\begin{array}{l}\text { Pelo menos } 3 \text { dias consecutivos } \\
\text { com temperaturas mínimas e } \\
\text { máximas concomitantemente } \\
\text { inferiores ao percentil } 10 \text { para } \\
\text { aquela estação do ano. }\end{array}$ \\
\hline OMM & $\begin{array}{l}\text { Cunha e Leal (2013); } \\
\text { Mateus (2014); } \\
\text { IPMA (2017) }\end{array}$ & $\begin{array}{l}T_{\min } \text { do ar / Muito } \\
\text { rigoroso }\end{array}$ & $\begin{array}{l}\text { Pelo menos } 6 \text { dias consecutivos } \\
\text { com temperatura mínima do ar } \\
\text { inferior em } 5^{\circ} \mathrm{C} \text { ao valor médio } \\
\text { das temperaturas mínimas } \\
\text { diárias no período de referência } \\
\text { (normais mensais) (1961- } \\
1990) \text {. }\end{array}$ \\
\hline
\end{tabular}




\begin{tabular}{|c|c|c|c|}
\hline $\begin{array}{l}\text { Índice do } \\
\text { IPCC - } \\
\text { adaptado }\end{array}$ & IPCC (2001) & $\begin{array}{l}T_{\min } \text { do ar / Muito } \\
\text { rigoroso }\end{array}$ & $\begin{array}{c}\text { Pelo menos } 5 \text { dias consecutivos } \\
\text { com temperatura mínima do ar } \\
\text { inferior em } 5^{\circ} \mathrm{C} \text { ao valor médio } \\
\text { das temperaturas mínimas } \\
\text { diárias no período de referência } \\
\text { (normais mensais) (1961- } \\
1990) \text {. }\end{array}$ \\
\hline $\begin{array}{l}\text { Nairn e } \\
\text { Fawcett } \\
(2013)\end{array}$ & $\begin{array}{l}\text { Nairn e Fawcett (2013); } \\
\text { Reboita et al. (2015) }\end{array}$ & $T_{\text {méd do ar / Rigoroso }}$ & $\begin{array}{l}\text { Pelo menos } 3 \text { dias consecutivos } \\
\text { com temperatura média do ar } \\
\left(T_{\text {méd }}=\left(T_{\text {máx }}-T_{\text {min }}\right) / 2\right) \text { abaixo } \\
\text { do percentil } 5 \text { da série histórica. }\end{array}$ \\
\hline $\begin{array}{l}\text { Firpo et al. } \\
\text { (2012) }\end{array}$ & Firpo et al. (2012) & $\begin{array}{c}T_{\min } \text { do ar / Pouco } \\
\text { rigoroso }\end{array}$ & $\begin{array}{c}\text { Pelo menos } 5 \text { dias consecutivos } \\
\text { com temperatura mínima } \\
\text { abaixo do valor normal } \\
\text { climatológico esperado } \\
\text { (anomalia) para cada dia do } \\
\text { ano em questão e para a } \\
\text { localidade em estudo. }\end{array}$ \\
\hline $\begin{array}{l}\text { Vavrus et } \\
\text { al. (2006) - } \\
\text { adaptado }\end{array}$ & $\begin{array}{c}\text { Vavrus et al. (2006); } \\
\text { Alves (2016); Silveira RB } \\
\text { (2016); Alves et al. } \\
\text { (2017) }\end{array}$ & $T_{\text {méd do ar / Rigoroso }}$ & $\begin{array}{c}\text { Pelo menos } 2 \text { dias consecutivos } \\
\text { com temperatura média do ar } \\
\text { abaixo de } 2 \text { desvio padrão ( } 10 \\
\text { de janeiro a } 31 \text { de dezembro), } \\
\text { abaixo da temperatura média } \\
\text { do ar no local e período } \\
\text { considerado. }\end{array}$ \\
\hline $\begin{array}{l}\text { Percentil } 5 \text { - } \\
\text { teste } \\
\text { aleatório }\end{array}$ & Testado pelos autores & $T_{\min }$ do ar / Rigoroso & $\begin{array}{c}\text { Pelo menos } 3 \text { dias consecutivos } \\
\text { com temperatura mínima } \\
\text { abaixo do percentil } 5 \text { daquela } \\
\text { estação do ano correspondente } \\
\text { ao período. }\end{array}$ \\
\hline
\end{tabular}

Adaptado de: Monteiro e Carvalho, 2013; Fante e Armond, 2016. Organizado pelos autores.

A Figura 1 mostra a localização dos três pontos (estações meteorológicas) para os quais os métodos foram testados, frente ao mapa hipsométrico do estado de SC. Os locais selecionados (Florianópolis (São José), Lages e Chapecó), possibilitam a averiguação de diferentes condições geográficas (latitude, altitude, relevo, vegetação, continentalidade e maritimidade), que podem inferir nos resultados obtidos de cada método. A continentalidade, a altitude e a maritimidade são fatores que possuem maior influência nos sistemas atmosféricos (MONTEIRO; MENDONÇA, 2014, p.5). 


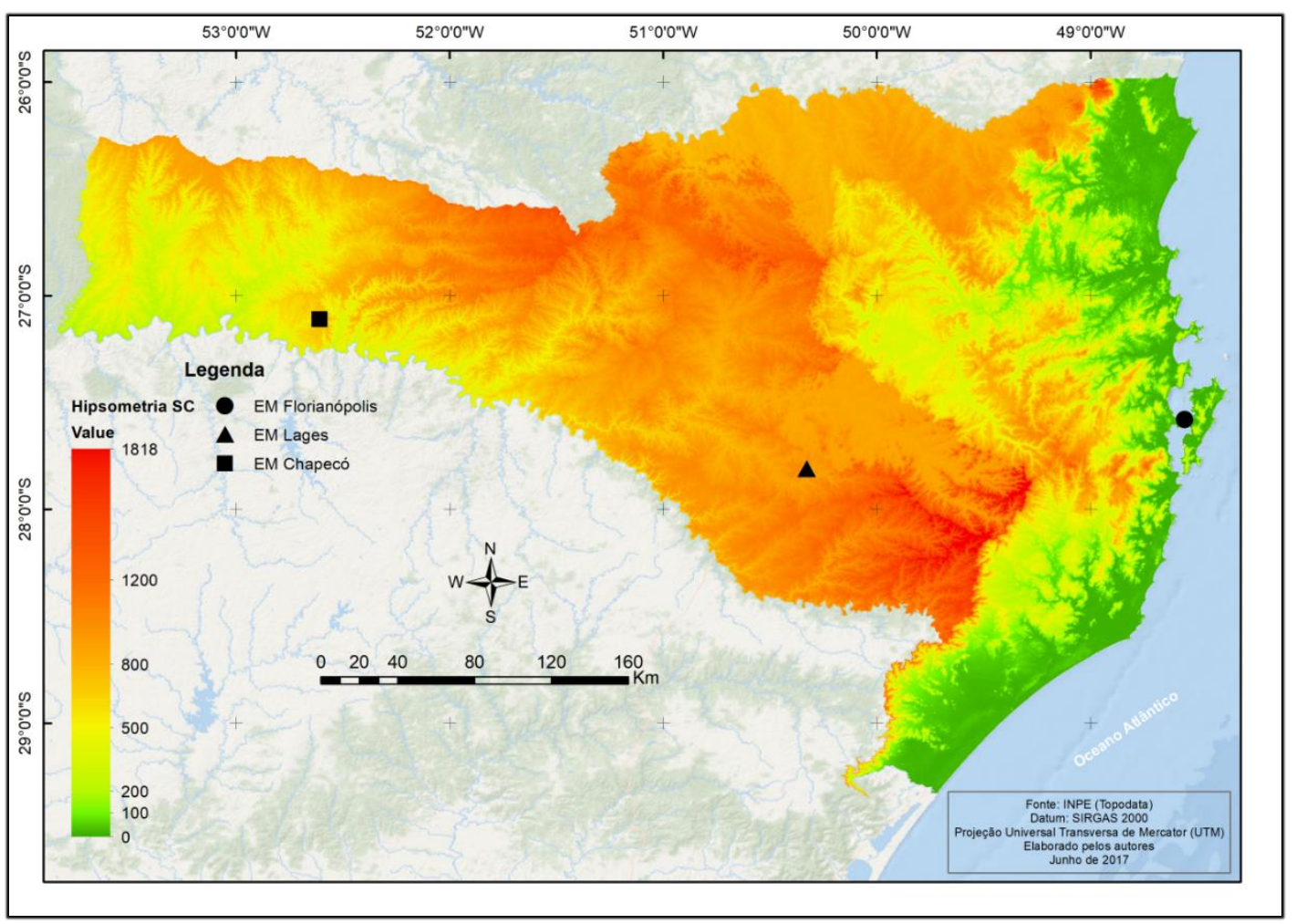

Figura 1 - Mapa hipsométrico (em metros) de SC com as estações meteorológicas (EM) utilizadas.

De acordo ainda com o Quadro 1, dos dez métodos analisados, cinco tiveram algumas adaptações em relação aos originais. O método nomeado neste trabalho de EE - Silveira (2016) considerou como OdF o que originalmente em seu artigo Silveira RD (2016) chamou de Evento Extremo, havendo, portanto, diferença apenas entre as nomenclaturas adotadas. Já o método nomeado como Tmáx e Tmín (10\%), em suas pesquisas bases citadas (YAGUE et al., 2006; LANDA, 2012) consideraram como OdF um determinado período com temperaturas mínimas e máximas concomitantemente inferiores ao percentil 10 para aquela estação do ano. Além destes autores, Silveira RD (2016) também utilizou a mesma análise, mas, a autora considerou três dias consecutivos como mínimo para detecção de uma OdF, a mesma os chamou de Supereventos, neste sentido, a presente pesquisa utilizou este mesmo período.

O método Índice do IPCC inicialmente foi utilizado para detecção de ondas de calor, aqui sua aplicação foi invertida para detectar OdF, mantendo o critério, todavia, considerando que uma OdF ocorre quando num período de cinco dias consecutivos ou mais, a temperatura mínima do ar é inferior em $5^{\circ} \mathrm{C}$ ao valor médio das temperaturas mínimas diárias no período de referência (normais climatológicas). O método de Vavrus et al., (2016) também foi adaptado, pois originalmente os autores aplicaram somente para o período invernal no hemisfério norte, no presente artigo as análises englobaram o ano todo.

O único teste aplicado sem ser embasado em alguma pesquisa específica foi o Percentil 5 - teste aleatório, sendo aqui apresentado como uma 
proposta deste estudo. Nesse caso, para detectar uma OdF é necessário que em três dias consecutivos ou mais as temperaturas mínimas estejam abaixo do percentil 5 daquela estação do ano correspondente ao período analisado/observado.

Os métodos analisados, para além da técnica estatística, também apresentam suas definições para onda de frio. Todos eles, conceitualmente, estão diretamente relacionados aos impactos socioambientais, como: na saúde, no bem-estar da população, na agricultura, em outros setores econômicos, podendo ocasionar prejuízos e até mesmo catástrofes. Os métodos, específicos ou não para cada tipo de diagnóstico, sempre buscam gerar análises capazes de mensurar e/ou prevenir impactos significantes.

\section{ANÁLISES ESTATÍSTICAS}

Os resultados de $\mathrm{n}^{\circ}$ de OdF detectados pelos dez métodos, por estação do ano, nas localidades analisadas, foram submetidos à Análise da Variância (ANOVA) Fatorial com nível de significância de $a=5 \%$, para verificar quais métodos são diferentes estatisticamente entre si. Como neste estudo estevese interessado em medir o efeito de mais de um fator sobre a variável de interesse optou-se pela ANOVA Fatorial.

Como houve diferença significativa entre os métodos de detecção de OdF pelo teste $F$, aplicou-se um teste Tukey de comparação múltipla de médias de ocorrências de OdF, ao nível de $a=5 \%$. Todas as análises foram realizadas no software STATISTICA $8.0 \AA$.

\section{RESULTADOS E DISCUSSÕES}

O no médio das OdF variou de acordo com o método analisado, com a estação do ano e em função do local (Tabela 1). Para os três locais analisados, os maiores registros médios de OdF foram detectados com a utilização do método de Firpo et al. (2012), com grande destaque em comparação com os demais. Como exemplo, no período invernal, Chapecó apresentou média de 3,91 OdF; já Lages, teve média de 2,91 e Florianópolis 2,55 OdF. Outro detalhe percebido com praticamente todos os métodos é a identificação de OdF em todas as estações do ano, inclusive, identificando um no médio de OdF maior em estações do ano climatologicamente menos frias. Nesse sentido, chama atenção, para Florianópolis, que o método de Firpo et al. (2012) detectou em média 3,55 OdF estivais. Basicamente, isso ocorre porque são utilizados parâmetros estatísticos referentes à estação do ano ou ao dia em análise. Fazendo isso, a OdF identificada é, na verdade, um evento frio extremo para a época do ano em análise, quando, naturalmente, os eventos de frio mais intensos ocorrem no inverno. 
Tabela 1 - No médio de OdF por estação do ano e interanual diante dos diferentes métodos analisados para: Florianópolis, Lages e Chapecó, no período de 2000 a 2010.

\begin{tabular}{|c|c|c|c|c|c|c|c|c|c|c|c|c|c|c|c|}
\hline \multirow{2}{*}{ Método } & \multicolumn{5}{|c|}{ Florianópolis } & \multicolumn{5}{|c|}{ Lages } & \multicolumn{5}{|c|}{ Chapecó } \\
\hline & Ver. & Out. & Inv. & Pri. & MI* & Ver. & Out. & Inv. & Pri. & MI* & Ver. & Out. & Inv. & Pri. & MI* \\
\hline $\begin{array}{l}\text { Vavrus et al. } \\
(2006)\end{array}$ & 0,18 & 0,45 & 1,09 & 0,45 & 2,18 & 0,09 & 0,27 & 1,91 & 0,45 & 2,73 & 0,09 & 0,45 & 2,82 & 0,82 & 4,18 \\
\hline $\begin{array}{l}\text { EE - Silveira } \\
(2016)\end{array}$ & 0,91 & 1,18 & 1,18 & 1,18 & 4,45 & 1,18 & 1,00 & 0,73 & 0,91 & 3,82 & 1,18 & 1,55 & 1,00 & 1,27 & 5,00 \\
\hline $\begin{array}{l}\text { Índice } \\
\text { Australiano }\end{array}$ & 0,09 & 0,91 & 0,45 & 0,64 & 2,09 & 0,18 & 0,82 & 0,45 & 0,36 & 1,82 & 0,55 & 0,82 & 0,45 & 0,73 & 2,55 \\
\hline CSDI - OMM & 0,00 & 0,36 & 0,09 & 0,18 & 0,64 & 0,00 & 0,09 & 0,09 & 0,09 & 0,27 & 0,00 & 0,18 & 0,00 & 0,27 & 0,45 \\
\hline $\begin{array}{l}\text { Percen. } 5 \text { - } \\
\text { teste aleatório }\end{array}$ & 0,27 & 0,55 & 0,36 & 0,64 & 1,82 & 0,18 & 0,55 & 0,18 & 0,36 & 1,27 & 0,55 & 0,36 & 0,18 & 0,64 & 1,73 \\
\hline $\begin{array}{l}\text { Tmáx e Tmín } \\
(10 \%)\end{array}$ & 0,00 & 0,36 & 0,09 & 0,18 & 0,64 & 0,45 & 0,36 & 0,18 & 0,18 & 1,18 & 0,09 & 0,55 & 0,18 & 0,55 & 1,36 \\
\hline OMM & 0,00 & 0,00 & 0,09 & 0,00 & 0,09 & 0,00 & 0,09 & 0,09 & 0,00 & 0,18 & 0,00 & 0,00 & 0,27 & 0,00 & 0,27 \\
\hline $\begin{array}{l}\text { Índice do } \\
\text { IPCC }\end{array}$ & 0,00 & 0,18 & 0,27 & 0,00 & 0,45 & 0,00 & 0,18 & 0,45 & 0,09 & 0,73 & 0,00 & 0,00 & 0,45 & 0,09 & 0,55 \\
\hline $\begin{array}{l}\text { Nairn e } \\
\text { Fawcett } \\
(2013)\end{array}$ & 0,00 & 0,00 & 1,73 & 0,27 & 2,00 & 0,00 & 0,18 & 2,00 & 0,18 & 2,36 & 0,00 & 0,09 & 1,64 & 0,18 & 1,91 \\
\hline $\begin{array}{l}\text { Firpo et al. } \\
(2012)\end{array}$ & 3,55 & 3,09 & 2,55 & 2,91 & 12,09 & 2,27 & 2,45 & 2,91 & 2,82 & 10,45 & 3,09 & 3,09 & 3,91 & 2,73 & 12,82 \\
\hline
\end{tabular}

*MI = Média Interanual dos 11 anos analisados.

Ao contrário do método de Firpo et al. (2012), os métodos OMM, Índice do IPCC e CSDI-OMM contabilizaram as menores médias nos três locais, tanto nas médias interanuais como por estações do ano, demonstrando grande rigor para detecção de uma OdF, muito em função do no de dias necessários para que uma onda seja classificada. Outro método que se destacou foi o EE Silveira (2016), com semelhança na recorrência média de OdF entre as estações do ano para Florianópolis, Lages e Chapecó, muito em virtude da técnica utilizada e da duração mínima necessária (3 dias).

Chama a atenção na Tabela 1 os métodos de Nairn e Fawcett (2013) e Vavrus et al. (2006), pois ambos apresentaram maiores no médios de ocorrência de OdF na estação invernal, possivelmente por utilizarem a temperatura média do ar e não considerarem muitos dias consecutivos para determinação de uma OdF. O Índice do IPCC também apresenta maior no médio no inverno, entretanto, são médias baixas. Vale ressaltar que o índice Tmáx e Tmíx (10\%) é o único a considerar duas variáveis na detecção das OdF (Tmáx e Tmín), suas médias durante as estações do ano foram baixas, com destaque nos três municípios para as estações de transição.

Quando aplicado a análise da variância (ANOVA) Fatorial para o número médio de ocorrências de OdF, verificaram-se diferenças estatísticas significativas ao nível de $5 \%$ para Local, Método, Estação do Ano e a interação entre Método x Estação do ano (Tabela 2). Não foi encontrada interação significativa entre Local x Método, Local x Estação do Ano e, Local x Método x Estação do Ano. 
Tabela 2 - Análise da Variância (ANOVA) Fatorial com nível de significância de $a=5 \%$.

\begin{tabular}{lrrrrr}
\hline Análise & \multicolumn{1}{c}{ SS } & Grau de liberdade & MS & \multicolumn{1}{c}{ F } & \multicolumn{1}{c}{-valor } \\
\hline Local & 5,2909 & 2 & 2,6455 & 5,409 & $0,004586^{*}$ \\
Método & 875,41 & 9 & 97,268 & 198,88 & $0^{*}$ \\
Estação do ano & 31,772 & 3 & 10,591 & 21,654 & $0 *$ \\
Local x Método & 13,2242 & 18 & 0,7347 & 1,502 & 0,080585 \\
Local x Estação do ano & 3,2485 & 6 & 0,5414 & 1,107 & 0,355964 \\
Método x Estação do ano & 120,993 & 27 & 4,4812 & 9,162 & $0 *$ \\
Local x Método x Estação do ano & 32,4182 & 54 & 0,6003 & 1,227 & 0,128458 \\
Error & 586,909 & 1200 & 0,4891 & & \\
\hline
\end{tabular}

Nota: os valores de $\mathrm{p}$ ( $\mathrm{p}$-valor) seguidos por $\left(^{*}\right)$ diferem entre si ao nível de significância de 5\%.

Na realização do teste de Tukey de comparação múltipla de médias, ao nível de significância de $a=5 \%$, verificou-se que o número de OdF nas localidades de Lages e Chapecó são diferentes estatisticamente, para o período de 2000 a 2010 (Tabela 3). Já Florianópolis não difere significativamente de Lages e de Chapecó. Este resultado não comprovou o que se esperava. Hipoteticamente esperava-se que Chapecó e Lages apresentassem similitude, ou, que os três locais fossem diferentes estatisticamente para esta análise; já que são regiões climatologicamente distintas.

A ocorrência do menor número de OdF em Lages, região de planalto, indica se tratar de uma região climatológica estável, onde as anomalias são menos frequentes. A região de Chapecó, por seu relevo e geomorfologia, continentalidade e posição geográfica recebe primeiro os impactos severos das entradas das massas de ar polares e/ou as frentes frias na maior parte do ano; podendo-se assim, explicar a ocorrência do maior $n^{\circ}$ de OdF. Alves (2016) ao investigar as OdF invernais em SC no período de 1983 a 2013 observou que o município de Chapecó foi aquele com maior ocorrência (93 eventos).

Não se encontrou uma explicação para Florianópolis ter a mesma conformidade estatística, quanto ao $\mathrm{n}^{\circ}$ médio de OdF, que Chapecó e Lages, visto serem regiões climatológicas distintas. Recomenda-se que estes resultados sejam melhores estudados em trabalhos futuros.

Tabela 3 - Valores médios de OdF para os locais analisados pelo teste Tukey ao nível de significância $a=5 \%$, no período de 2000-2010.

\begin{tabular}{lrcc}
\hline \multicolumn{1}{c}{ Local } & $N^{\circ}$ OdF - Média & 1 & 2 \\
\hline Lages & 0,620455 & $* * * *$ & \\
Florianópolis & 0,661364 & $* * * *$ & $* * * *$ \\
Chapecó & 0,770455 & & $* * * *$ \\
\hline
\end{tabular}

Com base na Tabela 4 verifica-se que o método de Firpo et al. (2012) difere de todos os outros métodos, ou seja, este índice detecta maior no de ondas. Utilizando tal método, com base nesta análise, em comparação com os demais, se espera que o $\mathrm{n}^{\circ}$ médio de OdF seja significativamente maior. Isto se deve ao fato de que este método detecta ondas duradouras, porém, pouco rigorosas visto que usa as anomalias da Tmín, facilitando com que a duração mínima para classificar uma onda seja atingida.

Outro método que divergiu estatisticamente de todos os demais foi o EE - Silveira (2016), que apresentou número médio de OdF inferior somente ao método de Firpo et al. (2012), possivelmente devido ao método adotado. Firpo et al. (2012) necessita que a Tmín observada nos dias da OdF sejam inferiores 
aos limiares diários encontrados ao longo da série baseados na média aritmética de cada dia; ao passo que o método de EE - Silveira (2016) utiliza o percentil 10 de cada estação do ano, o que torna seus limiares mais rigorosos comparados com o de Firpo et al. (2012), por mais que o no mínimo de dias para se detectar uma OdF seja menor.

Não houve diferença estatística entre os métodos de Vavrus et al. (2006), do Índice Australiano e Nairn e Fawcett (2013), que por sua vez diferem dos outros sete. Utilizando qualquer um dos três métodos - Vavrus et al. (2006), Índice Australiano e Nairn e Fawcett (2013) - espera-se obter resultados similares.

O Índice Australiano tem média de OdF estatisticamente igual aos métodos de Nair e Fawcett (2013) e Percen. 5 (Tabela 4), podendo isto estar relacionado tanto com a duração (dias) considerada para identificar uma OdF como com a técnica estatística. Ao passo que o Índice Australiano considera no mínimo quatro dias consecutivos com a Tmín abaixo do percentil 10 encontrado para a estação do ano, os outros dois métodos utilizam o percentil 5 com três dias consecutivos no mínimo. De certa forma, o rigor maior no no de dias para classificação da OdF no Índice Australiano é compensando pelo maior rigor estatístico dos outros dois, que são mais restritos devido ao uso do percentil 5.

São estatisticamente iguais os métodos de Nair e Fawcett (2013), Percen. 5 e Tmáx e Tmín (10\%), todos utilizam três dias como duração mínima para uma OdF ser detectada e isto pode explicar a igualdade estatística. Porém, os demais critérios de cada método não são totalmente idênticos, divergem em alguns pontos, por exemplo: um utiliza Tméd, outro Tmín e um deles com a Tmín e a Tmáx de forma conjunta. Subjetivamente, espera-se que a utilização da Tméd seja mais adequada que a Tmín em regiões subtropicais, onde, principalmente no outono e primavera, ocorrem dias com valores baixos de Tmín e valores altos de Tmáx. Portanto, ao ser utilizado os extremos de Tméd, espera-se eliminar da análise os casos em que o frio ocorre apenas em parte do dia por causa da grande amplitude térmica.

Os valores médios de OdF do método Percen. 5 são iguais estatisticamente aos do Tmáx e Tmín (10\%) e do Índice do IPCC, contudo, nota-se considerável diferença entre $0 \mathrm{n}^{\circ}$ de OdF médio do Percen. 5 $(0,401515)$ para o Índice do IPCC $(0,143939)$ (Tabela 4). Observando as técnicas e parâmetros de cada método, não há muita semelhança entre os mesmos.

Os métodos Tmáx e Tmín (10\%), Índice do IPCC, CSDI-OMM e OMM também são iguais estatisticamente entre si, estes foram os que apresentaram menores médias de OdF entre todos. Os quatro apresentam excepcionalidade, servindo para encontrar eventos rigorosos. Monteiro e Carvalho (2013) apontaram o mesmo para os métodos da OMM e o CSDI-OMM. Excluindo o método Tmáx e Tmín (10\%) é possível afirmar que os outros três são adequados para encontrar eventos extremos de longa duração. 
Tabela 4 - Valores médios de OdF entre os métodos de detecção pelo teste Tukey ao nível de significância $a=5 \%$, para Florianópolis, Lages e Chapecó, no período de 2000 2010.

\begin{tabular}{lrrrrrrrr}
\hline \multicolumn{1}{c}{ Método } & $\mathrm{N}^{\circ}$ OdF - Médio & 1 & 2 & 3 & 4 & 5 & 6 & 7 \\
\hline OMM & 0,045455 & $* * * *$ & & & & & & \\
CSDI - OMM & 0,113636 & $* * * *$ & & & & & \\
Índice do IPCC & 0,143939 & $* * * *$ & $* * * *$ & & & & \\
Tmáx e Tmín (10\%) & 0,265152 & $* * * *$ & $* * * *$ & $* * * *$ & & & \\
Percen. 5 - teste aleatório & 0,401515 & & $* * * *$ & $* * * *$ & $* * * *$ & & & \\
Nairn e Fawcett (2013) & 0,522727 & & & $* * * *$ & $* * * *$ & $* * * *$ & & \\
Índice Australiano & 0,537879 & & & & $* * * *$ & $* * * *$ & & \\
Vavrus et al. (2006) & 0,757576 & & & & & $* * * *$ & & \\
EE - Silveira (2016) & 1,106061 & & & & & & $* * * *$ & \\
Firpo et al. (2012) & 2,94697 & & & & & & & $* * * *$ \\
\hline
\end{tabular}

Na Tabela 5 pode-se observar os resultados do número médio de OdF nas diferentes estações do ano. Verifica-se que não houve diferença estatística na ocorrência de OdF entre as estações de transição (primavera e outono); porém, diferente do verão e inverno. Já o verão apresentou o menor no médio de OdF, diferindo estatisticamente do inverno, que apresentou a maior ocorrência. Esse resultado era esperado dentro dos aspectos climáticos de SC. Conforme Grimm (2009) a situação geográfica da Região Sul brasileira, no subtrópico, garante a maior amplitude térmica no ciclo anual, com maior diferença entre inverno e verão. Esta região é uma das pouquíssimas do Brasil com precipitação nival, podendo registrar temperaturas consideravelmente frias.

Tabela 5 - Valores médios de OdF para as estações do ano para Florianópolis, Lages e Chapecó, no período de 2000-2010, pelo teste Tukey ao nível de significância $a=5 \%$.

\begin{tabular}{lrrcc}
\hline Estação do ano & $N^{\circ}$ OdF - Média & 1 & 2 & 3 \\
\hline Verão & 0,49697 & $* * * *$ & \\
Primavera & 0,639394 & $* * * *$ & & \\
Outono & 0,672727 & $* * * *$ & & \\
Inverno & 0,927273 & & & $* * * *$ \\
\hline
\end{tabular}

A Tabela 6 apresenta os resultados da análise de interação entre métodos de identificação de OdF e estações do ano. Observa-se na tabela que entre o Índice do IPCC na estação de verão (1 $1^{a}$ linha) até o Índice Australiano na primavera (29a linha), todos os métodos tiveram similaridade estatística nas estações do ano. Vale indicar que para a estação de verão os métodos Índice do IPCC, OMM, Nairn e Fawcett (2013) e CSDI-OMM, além da estação de primavera no método OMM, não registraram OdF.

O método de Firpo et al. (2012) tem similaridade estatística igual nas quatro estações e não possui semelhança em relação as estações do ano dos demais métodos, demonstrando singularidade para esta avaliação. Da mesma forma baseado nas estações do ano, o método EE - Silveira (2016) possui igualdade estatística nas estações do ano, mas, também possui igualdade com outras estações do ano de outros métodos, não é "isolado" como o de Firpo et al. (2012).

O método de Vavrus et al. (2006) no inverno diverge das demais estações, ou seja, ao passo que o verão, outono e primavera são iguais 
estatisticamente, a estação invernal não. O mesmo ocorre com as demais estações do método Nairn e Fawcett (2013). Destaca-se que no inverno estes dois métodos são similares estatisticamente.

O Índice Australiano para o outono não demonstrou grande diferença no número médio de OdF quando comparado com a primavera, entretanto, não são iguais estatisticamente.

Tabela 6 - Comparação múltipla de médias de ondas de frio (OdF) pelo teste Tukey ao nível de significância $a=5 \%$. Métodos de deteç̧ão de ondas versus estação do ano para Florianópolis, Lages e Chapecó, no período de 2000-2010.

\begin{tabular}{|c|c|c|c|c|c|c|c|c|c|c|c|}
\hline Método & Estação do ano & $\mathrm{N}^{\circ}$ OdF - Média & 1 & 2 & 3 & 4 & 5 & 6 & 7 & 8 & 9 \\
\hline Índice do IPCC & Verão & 0 & $* * * *$ & & & & & & & & \\
\hline OMM & Verão & 0 & $* * * *$ & & & & & & & & \\
\hline Nairn e Fawcett (2013) & Verão & 0 & $* * * *$ & & & & & & & & \\
\hline OMM & Primavera & 0 & $* * * *$ & & & & & & & & \\
\hline CSDI - OMM & Verão & 0 & $* * * *$ & & & & & & & & \\
\hline OMM & Outono & 0,030303 & $* * * *$ & & & & & & & & \\
\hline Índice do IPCC & Primavera & 0,060606 & $* * * *$ & & & & & & & & \\
\hline CSDI - OMM & Inverno & 0,060606 & $* * * *$ & & & & & & & & \\
\hline Nairn e Fawcett (2013) & Outono & 0,090909 & $* * * *$ & & & & & & & & \\
\hline Vavrus et al. (2006) & Verão & 0,121212 & $* * * *$ & & & & & & & & \\
\hline Índice do IPCC & Outono & 0,121212 & $* * * *$ & & & & & & & & \\
\hline OMM & Inverno & 0,151515 & $* * * *$ & & & & & & & & \\
\hline Tmáx e Tmín (10\%) & Inverno & 0,151515 & $* * * *$ & & & & & & & & \\
\hline Tmáx e Tmín (10\%) & Verão & 0,181818 & $* * * *$ & $* * * *$ & & & & & & & \\
\hline CSDI - OMM & Primavera & 0,181818 & $* * * *$ & $* * * *$ & & & & & & & \\
\hline Nairn e Fawcett (2013) & Primavera & 0,212121 & $* * * *$ & $* * * *$ & & & & & & & \\
\hline CSDI - OMM & Outono & 0,212121 & $* * * *$ & $* * * *$ & & & & & & & \\
\hline Percen. 5 - teste aleatório & Inverno & 0,242424 & $* * * *$ & $* * * *$ & & & & & & & \\
\hline Índice Australiano & Verão & 0,272727 & $* * * *$ & $* * * *$ & & & & & & & \\
\hline Tmáx e Tmín (10\%) & Primavera & 0,30303 & $* * * *$ & $* * * *$ & $* * * *$ & & & & & & \\
\hline Percen. 5 - teste aleatório & Verão & 0,333333 & $* * * *$ & $* * * *$ & $* * * *$ & & & & & & \\
\hline Vavrus et al. (2006) & Outono & 0,393939 & $* * * *$ & $* * * *$ & $* * * *$ & & & & & & \\
\hline Índice do IPCC & Inverno & 0,393939 & $* * * *$ & $* * * *$ & $* * * *$ & & & & & & \\
\hline Tmáx e Tmín (10\%) & Outono & 0,424242 & $* * * *$ & $* * * *$ & $* * * *$ & $* * * *$ & & & & & \\
\hline Índice Australiano & Inverno & 0,454545 & $* * * *$ & $* * * *$ & $* * * *$ & $* * * *$ & $* * * *$ & & & & \\
\hline Percen. 5 - teste aleatório & Outono & 0,484848 & $* * * *$ & $* * * *$ & $* * * *$ & $* * * *$ & $* * * *$ & & & & \\
\hline Percen. 5 - teste aleatório & Primavera & 0,545455 & $* * * *$ & $* * * *$ & $* * * *$ & $* * * *$ & $* * * *$ & & & & \\
\hline Vavrus et al. (2006) & Primavera & 0,575758 & $* * * *$ & $* * * *$ & $* * * *$ & $* * * *$ & $* * * *$ & $* * * *$ & & & \\
\hline Índice Australiano & Primavera & 0,575758 & $* * * *$ & $* * * *$ & $* * * *$ & $* * * *$ & $* * * *$ & $* * * *$ & & & \\
\hline Índice Australiano & Outono & 0,848485 & & $* * * *$ & $* * * *$ & $* * * *$ & $* * * *$ & $* * * *$ & & & \\
\hline EE - Silveira (2016) & Inverno & 0,969697 & & & $* * * *$ & $* * * *$ & $* * * *$ & $* * * *$ & & & \\
\hline EE - Silveira (2016) & Verão & 1,090909 & & & & $* * * *$ & $* * * *$ & $* * * *$ & & & \\
\hline EE - Silveira (2016) & Primavera & 1,121212 & & & & & $* * * *$ & $* * * *$ & $* * * *$ & & \\
\hline EE - Silveira (2016) & Outono & 1,242424 & & & & & & $* * * *$ & $* * * *$ & & \\
\hline Nairn e Fawcett (2013) & Inverno & 1,787879 & & & & & & & $* * * *$ & $* * * *$ & \\
\hline Vavrus et al. (2006) & Inverno & 1,939394 & & & & & & & & $* * * *$ & \\
\hline Firpo et al. (2012) & Primavera & 2,818182 & & & & & & & & & $* * * *$ \\
\hline Firpo et al. (2012) & Outono & 2,878788 & & & & & & & & & $* * * *$ \\
\hline Firpo et al. (2012) & Verão & 2,969697 & & & & & & & & & $* * * *$ \\
\hline Firpo et al. (2012) & Inverno & 3,121212 & & & & & & & & & $* * * *$ \\
\hline
\end{tabular}

\section{CONCLUSÕES}

Os métodos de detecção de ondas de frio variam, estatisticamente, em função do local e da época do ano; e, apresentam interações com as estações do ano. Há diferença estatística entre as localidades de Lages e Chapecó quanto à ocorrência das OdF; porém, o mesmo não se pode dizer de Florianópolis em relação à Lages e Chapecó.

Não há na bibliografia um consenso sobre qual método deva ser utilizado para identificação de OdF. Todos os 10 métodos aqui analisados identificam as OdF quando a temperatura (Tmín, Tméd ou Tmín + Tmáx) é menor que um determinado valor abaixo de um limiar específico. Entretanto, 
as definições desses limites assim como o tempo de duração em que esses limites são ultrapassados mudam de um método para outro.

O rigor do método é determinante para o no de OdF identificadas. Esse rigor é estabelecido pelo tamanho da anomalia abaixo da média considerada como limiar e, também, pelo $n^{\circ}$ de dias mínimo definido para ser uma OdF. Considerando esses dois aspectos, o método menos rigoroso (que identificou o maior no de OdF) é o de Firpo et al. (2012) que, apesar de assumir uma duração mínima de cinco dias consecutivos, estabelece que qualquer valor abaixo da média climatológica no dia observado é válido para identificar uma OdF. Os métodos de detecção de OdF tiveram resultados distintos. Pode-se destacar que o método de Firpo et al. (2012) foi o que apresentou destacada diferença, conforme supracitado, seguido pelo método de EE - Silveira (2016). Estes dois métodos foram os que apresentaram o maior $n^{\circ}$ de OdF, respectivamente.

Por outro lado, o método mais rigoroso é o da OMM, que exige seis dias com a temperatura mínima do dia abaixo do limite dado pela temperatura climatológica menos um valor fixo de $5^{\circ} \mathrm{C}$. Os métodos da OMM, o CSDI-OMM, o Índice do IPCC e o Tmáx e Tmín (10\%) tiveram similitude e podem ser considerados métodos muito rigorosos na detecção das ondas, visto que tiveram baixo $\mathrm{n}^{\circ}$ médio de OdF durante $\mathrm{o}$ ano e para todas as regiões analisadas.

Pode-se dizer ainda que os métodos de Vavrus et al. (2006), o do Índice Australiano e de Nairn e Fawcett (2013) são semelhantes; porém, os método do Índice Australiano e de Nairn e Fawcett (2013) não diferem do método Percentil 5 - teste aleatório.

A maior parte dos métodos aqui analisados identificam OdF ao longo de todo o ano. Isso ocorre pelo fato dos parâmetros estatísticos (principalmente a média climatológica) serem calculados com base no dia ou no trimestre analisado (estação do ano). Assim, são identificadas OdF que poderíamos chamar de eventos frios para aquela época do ano, mas que não necessariamente apresentem uma intensidade extrema de frio, apesar da subjetividade do que é frio e para quem.

Os fatores geográficos são determinantes no aspecto climatológico da localidade para a qual se pretende identificar as OdF. Ou seja, a utilização de um único método em duas localidades de diferentes características acarretará números diferentes de OdF identificadas.

Por mais que não seja o objetivo central desta pesquisa, entende-se que a determinação de qual método utilizar depende essencialmente da aplicação do estudo. Ou seja, se soubermos qual é o impacto na sociedade que queremos quantificar, ficará mais fácil de definir qual a variável climática, quais os limiares possíveis (e para qual período será considerada) e qual a duração que o evento deverá ter. Este trabalho apontou caminhos para que se possa tomar algumas decisões sobre qual (is) método (s) utilizar caso se queira encontra um no baixo, médio ou elevado de OdF, dependendo do que se pretende.

Acredita-se que ainda não há um método padrão para identificação de extremos de temperatura (onda de frio e de calor) ideal ou aplicável para todo o país, diante dos impactos para a saúde humana, para a agricultura, para o 
turismo ou para estes conjuntamente e demais setores. Porém, esta é uma necessidade existente em nível de organização para o Brasil, por mais que o país tenha dimensões continentais; pretende-se que este seja o próximo passo a tentar ser alcançado.

\section{AGRADECIMENTOS}

Os autores agradecem ao Laboratório de Climatologia Aplicada (LabClima/UFSC) e ao Programa de Pós-Graduação em Geografia (PPGG/UFSC). Esta é uma pesquisa vinculada ao projeto "Climas regionais e locais em Santa Catarina e América do Sul: mudanças, variabilidade, eventos extremos e impactos socioambientais", idealizado pela Prof.a Dr.a Magaly Mendonça (in memoriam). O $2^{\circ}$ autor agradece ainda à Coordenação de Aperfeiçoamento de Pessoal de Nível Superior (CAPES) por sua bolsa de doutorado (Processo no: 1696632).

\section{REFERÊNCIAS BIBLIOGRÁFICAS}

ALVES, M.P.A. Ondas de frio invernais em Santa Catarina e suas relações com as variabilidades climáticas de baixa frequência. Dissertação (Mestrado em Geografia) - Departamento de Geociências, Centro de Filosofia e Ciências Humanas, Universidade Federal de Santa Catarina. Florianópolis, 158 p.; 2016.

ALVES, M.P.A.; BRITO SILVEIRA, R.; BOLIGON MINUZZI, R.; ELVINO FRANKE, $A$. The Influence of the Antarctic Oscillation (AAO) on cold waves and occurrence of frosts in the state of Santa Catarina, Brazil. Climate, v.5, 13 p., 2017.

ALVES, M.P.A.; SILVEIRA, R.B.; FRANKE, A.E. Correlação das ondas de frio invernais e as ocorrências de geada no município de Caçador, Santa Catarina, Brasil. In: Anais do VI Simpósio Internacional de Climatologia (VI SIC), Natal RN, $2015 . \quad$ Disponível em: <http://www.labclima.ufsc.br/files/2010/04/Correla\%>. Acesso em: 23 ago. 2017.

BORSATO, V.A. A dinâmica climática do Brasil e massas de ares. $1^{a}$ ed. Curitiba, PR: CRV, 2016. 184 p.

BUREAU of METEOROLOGY. About the climate extremes analyses, 2017. Disponível em: <http://www.bom.gov.au/climate/change/about/extremes.shtml>. Acesso em: 12 jun. 2017.

CARSON, C.; HAJAT, S.; ARMSTRONG, B.; WILKINSON, P. Declining vulnerability to temperature-related mortality in London over the 20th century. American Journal of Epidemiology, v. 164, n. 1, p. 77-84, 2006.

CASTRO, A.L.C.; CALHEIROS, L.B.; CUNHA, M.I.R; BRINGEL, M.L.N.C. Manual de Desastres - Desastres Naturais. Ministério da Integração Nacional - MI, Brasília, v. 1, 2003.

CRED - Centre for Research on the Epidemiology of Disasters. EMDAT: International Disaster Database. 2015. Disponível em: <http://www.emdat.be/database>. Acesso em: 06 out. 2017. 
CUNHA, L.; LEAL, C. Ondas de calor e ondas de frio no município de Torres Novas. Cadernos de Geografia, n.32, p. 81-93, 2013.

ESCOBAR, G.C.J. Padrões sinóticos associados a ondas de frio na cidade de São Paulo. Revista Brasileira de Meteorologia, v.22, n.2, 241-254, 2007.

FANTE, K.P.; ARMOND, N.B. Ondas de frio e enfermidades respiratórias: análise na perspectiva da vulnerabilidade climática. Revista do Departamento de Geografia, n. spe, p. 145-159, 2016.

FIRPO, M.A.F.; SANSIGOLO, C.A.; de ASSIS, S.V. Climatologia e variabilidade sazonal do número de ondas de calor e de frio no Rio Grande do Sul associadas ao ENOS. Revista Brasileira de Meteorologia, v.27, p. 95-106, 2012.

FRANKE, A.E.; ALVES, M.P.A.; SILVEIRA, R.B. Probabilidade de ocorrência de ondas de frio estivais na costa catarinense. In: Anais do VI Simpósio Internacional de Climatologia (VI SIC), Natal - RN, 2015. Disponível em: <http://www.labclima.ufsc.br/files/2010/04/OdF_ver\%>. Acesso em: 23 ago. 2017.

GASPARRINI, A.; GUO, Y.; HASHIZUME, M.; LAVIGNE, E.; ZANOBETTI, A.; SCHWARTZ, J.; TOBIAS, A.; TONG, S.; ROCKLÖV, J.; FORSBERG, B.; LEONE, M.; SARIO, M. BELL, M.L.; GUO, Y.L.; WU, C.; KAN, H.; YI, S.; COELHO, M.S.Z.S.; SALDIVA, P.H.N.; HONDA, Y.; KIM, H.; ARMSTRONG, B. Mortality risk attributable to high and low ambient temperature: a multicountry observational study. The Lancet, p. 1-7, 2015.

GRIMM, A.M. Clima da região sul do Brasil. In: Tempo e Clima no Brasil. Iracema F.A.Cavalcanti [et al.] organizadores. São Paulo: Oficina de Textos, 463p., 2009.

IPCC - Intergovernamental Panel on Climate Change. Climate change 2013: the physical science basis. Summary for policymakers, technical summary and frequently asked questions. (Org) T.F., STOCKER; D., QIN.; G.K., PLATTNER; M., TIGNOR; S.K., ALLEN; J., BOSCHUNG; P.M., MIDGLEY. Working group I contribution to the IPCC Fifth Assessment Report (AR5). Cambridge Univ, Press, New York, 2013. Disponível em: <http://www.ipcc.ch/pdf/assessmentreport/ar5>. Acesso em: 14 jun. 2017.

IPCC - Intergovernmental Panel on Climate Change. Climate Change 2001: The Scientific Basis. Contribution of Working Group I to the Third Assessment Report of the Intergovernmental Panel on Climate Change [Houghton, J.T., Y. Ding, D.J. Griggs, M. Noguer, P.J. van der Linden, X. Dai, K. Maskell, and C.A. Johnson (eds.)]. Cambridge University Press, Cambridge, United Kingdom and New York, NY, USA, 881pp, 2001.

IPMA - Instituto Português do Mar e da Atmosfera. Glossários - Glossário Climatológico/Meteorológico, $2017 . \quad$ Disponível em: <https://www.ipma.pt/pt/educativa/glossario>. Acesso em: 12 jun. 2017.

LANDA, A.L.G. Olas de frío en la zona central del Estado de Veracruz. Facultad de Instrumentación Electrónica y Ciencis Atmosféricas. Universidad Veracruzana. 49 p., 2012.

MATEUS, C. Ondas de calor e ondas de frio em Coimbra: impactes na mortalidade da população. Dissertação de mestrado em Geografia Física, Universidade de Coimbra, 112 p., 2014. 
MENDONÇA, M.; ROMERO, H. Ondas de frio, índices de oscilação e impactos socioambientais das variabilidades climáticas de baixa frequência na América do Sul. Revista Acta Geográfica (UFRR), v.2, p.185-185, 2012.

MONTEIRO, A.; CARVALHO, V. Uma abordagem metodológica para avaliação de eventos climáticos extremos. In: AMORIM, M.C.T. de.; SANT'ANNA NETO, J.L.; MONTEIRO, A. (org). Climatologia urbana e regional: questões teóricas e estudos de caso. $1^{\text {a }}$ edição. São Paulo: Outras expressões - Geografia em Movimento, p. 117 - 142, 2013.

MONTEIRO, C.A.F. O clima da região Sul. Geografia do Brasil, Grande Região Sul. Rio de Janeiro: IBGE, p. 117-169, 1963.

MONTEIRO, M. Caracterização climática do estado de Santa Catarina: uma abordagem dos principais sistemas atmosféricos que atuam durante 0 ano. Revista Geosul, Florianópolis, v. 16, no 31, p 69-78, 2001.

MONTEIRO, M.A.; MENDONÇA, M. Dinâmica atmosférica do estado de Santa Catarina. In: HERRMANN, M. L. P. (org). Atlas de Desastres Naturais do Estado de Santa Catarina: período de 1980 a 2010. 2. ed. atual. e rev. - Florianópolis: IHGSC/Cadernos Geográficos, 219 p., 2014.

MORABITO, M.; CRISCI, A.; GRIFONI, D.; ORLANDINI, S.; CECCHI, L. ; BACCI, L.; MODESTI, P.; GENSINI, G.; MARACCHI, G. Winter air-mass-based synoptic climatological approach and hospital admissions for myocardial infarction in Florence, Italy. Environmental Research, n.102, pp.52-60, 2006.

MURARA, P.G.; COELHO, M.S.Z S.; AMORIM, M.C.C.T. Análise da influência meteorológica nas internações por doenças cardiovasculares. Caderno Prudentino de Geografia, v. 1, n. 32, p. 53- 65, 2010.

MURARA, P.G.; FUENTES, M.V. Neve. In: HERRMANN, M. L. P. (org). Atlas de Desastres Naturais do Estado de Santa Catarina: período de 1980 a 2010. 2. ed. atual. e rev. - Florianópolis: IHGSC/Cadernos Geográficos, 219 p., 2014.

NAIRN, J.; FAWCETT, R. Defining heatwaves: heatwave defined as a heatimpact event servicing all community and business sectors in Australia. CAWCR Technical Report no. 060, 2013.

NIMER, E. Climatologia do Brasil. Rio de Janeiro: IBGE, 1979, 422 p.

OMM - Organização Meteorológica Mundial. Climate Indices and Analysis for Sectoral Application. Italy, 53 p., 2011. Disponível em: <http://indico.ictp.it/event/a10167/session/16/contribution>. Acesso em: 12 jun. 2017.

REBOITA, M.S.; ESCOBAR, G.; LOPES, V.S. Climatologia sinótica de eventos de ondas de frio sobre a região sul de Minas Gerais. Revista Brasileira de Climatologia, v.16, p.72-92, 2015.

SILVEIRA, R.B. Ondas de frio em São Joaquim - Santa Catarina - Brasil: a saúde como fator dependente da qualidade de vida. Dissertação (Mestrado em Geografia) - Departamento de Geociências, Centro de Filosofia e Ciências Humanas, Universidade Federal de Santa Catarina. Florianópolis, 172 p., 2016.

SILVEIRA, R.B.; ALVES, M.P.A.; BITENCOURT, D.P. Extremos de temperatura e saúde pública: uma proposta para sistema de alerta de ondas de frio e calor. CADERNOS GEOGRÁFICOS (UFSC), v. 36, p. 49-62, 2017. 
SILVEIRA, R.B.; ALVES, M.P.A.; FRANKE, A.E. Internações por pneumonia frente às ondas de frio invernais na costa norte de Santa Catarina, Brasil. In: Anais do VI Simpósio Internacional de Climatologia (VI SIC), Natal - RN, 2015. Disponível em: <http://www.labclima.ufsc.br/files/2010/04/>. Acesso em: 23 ago. 2017.

SILVEIRA, R.D. Risco climático e vulnerabilidade socioespacial: o exemplo dos eventos extremos relacionados ao calor e ao frio. Revista Brasileira de Climatologia, v.19, p. 36-53, 2016.

TOBÍAS, A.; ARMSTRONG, B.; GASPARRINI, A. Brief Report: investigating uncertainty in the minimum mortality temperature: methods and application to 52 Spanish cities. Epidemiology, v. 28, n. 1, p. 72-76, 2017. Disponível em: <https://www.ncbi.nlm.nih.gov/pmc/articles/PMC5380105>. Acesso em: 14 jun. 2017.

VAVRUS, S.; WALSH, J.E.; CHAPMAN, W.L.; PORTIS, D. The behavior of extreme cold air outbreaks under greenhouse warming. International Journal of Climatology, n. 26, p. 1133-1147, 2006.

YAGÜE, C.; MARTIJA, M.; TORRES, J.; MALDONADO, A.; ZURITA, E. Análisis estadístico de las olas de calor y frío en España. In: XXIX Jornadas científicas de la AME. Aplicaciones Meteorológicas, 6 p. ISBN-13:979-84-611-1490-0, 2006. 